\title{
3-D Grid-Based Localization Technique in Mobile Sensor Networks
}

\author{
Jia Li ${ }^{1}$, Lei Sun ${ }^{1}$, Wai Yee Leong ${ }^{2}$, Peter H J Chong ${ }^{1}$ \\ ${ }^{1}$ School of Electrical and Electronic Engineering, Nanyang Technological University, Singapore \\ ${ }^{2}$ Singapore Institute of Manufacturing Technology, 71 Nanyang Drive, Singapore \\ E-mail: ehjchong@ntu.edu.sg \\ Received August 10, 2010; revised September 21, 2010; accepted October 25, 2010
}

\begin{abstract}
Considering the environmental protection, forest fire becomes a more and more serious problem and requires more concerns. This paper provides an efficient method for fire monitoring and detection in forests using wireless sensor network technology. The proposed technique estimates the location of a sensor node based on the current set of hop-count values, which are collected through the anchor nodes' broadcast. Our algorithm incorporates two salient features; grid-based output and event-triggering mechanism, to improve the accuracy while reducing the power consumption. Through the computer simulation, the output region obtained from our algorithm can always cover the target node. In addition, the algorithm was implemented and tested with a set of Crossbow sensors. Experimental results demonstrated the high feasibility and worked well in real environment.
\end{abstract}

Keywords: Wireless Mobile Sensor Networks, Forest Fire Detection, Localization Technique

\section{Introduction}

Wireless Sensor Networks (WSNs) provide unprecedented opportunities for monitoring areas of interests such as chemical factory, homes and offices, with lowcost, low-power and multi-functional sensors. As such, WSNs attract considerable amount of attention from researchers all over the world. Usually one should use a large number of sensor nodes to deploy a WSN because these sensors generally are small in size and can only communicate within short distances. Information can be collected from a WSN node through the base station. However, the collected information would be meaningless if we could not determine the location of a WSN node. Consequently, fast, efficient and low-cost localization techniques are highly desirable for WSNs applications.

The key idea of WSN localization is to allow some sensor nodes to know their own location at all time. Such nodes, usually called anchors, may be equipped with Global Positioning System (GPS) or be fixedly placed at pre-determined positions with known coordinates. For the sake of low cost, most sensor nodes do not know their locations. These nodes with unknown location information are called non-anchor nodes. Interestingly, their locations can be estimated by applying WSN local- ization techniques [1].

Localization techniques in WSNs are classified into two groups: range-based and range-free techniques. Range-based techniques use sophisticated hardware to conduct complex measurements on distance or angle of signal arrival to obtain location estimates. Typical range -based localization schemes includes those using received signal strength (RSS) [2], time of arrival (TOA) [3], angle of arrival (AOA) [4], and time difference of arrival (TDOA) [5]. Noteworthily, range-based localization techniques are applicable only when the non-anchor node of interest is within communication range of the anchor nodes. Due to the expensive hardware requirement, range-based techniques are generally considered as high-cost solutions. Consequently, this shortcoming unfortunately hinders them from being applied for forest fire surveillance, which is normally formed by millions of sensor nodes.

Range-free algorithms estimate the location of a sensor only based on the connectivity between non-anchor nodes and anchors. Three typical existing range-free techniques are the DV-hop [6], Monte-Carlo Localization (MCL) [7] and Monte-Carlo Box (MCB) [8] algorithms. The general principle of these techniques is that localization can be estimated from the proximity constraints, which are defined by a sensor node of interest 
being in the transmission ranges of other sensor nodes. For the authors' best knowledge, none of aforementioned localization techniques is exclusively designed forest fire surveillance. Consequently, when the existing localization techniques were applied in forest fire surveillance systems, they would inevitably result in certain disadvantages such as high complexity, low efficiency and large power consumption.

This paper aims to contribute a novel localization technique for forest fire surveillance by monitoring and tracking groups of animals using WSN technology. The proposed technique based on the existing range-free techniques and improves the accuracy. In brief, we propose to attach sensor nodes to selected animals. Whenever the temperature sensed at these animals' proximity rises beyond a predefined threshold, the localization module in the sensor would be activated and the subjects' motion paths are analyzed. The region of forest fire is estimated based on two indicators: (1) a group of animals are observed to run away from a certain area, and (2) the temperature sensed around the animals' surrounding environment is higher than a predefined threshold.

The rest of this paper is organized as follows. Section 2 revisits the related localization algorithms and Section 3 presents our proposed localization algorithm. In Section 4, the simulation results are discussed, which are followed by the experimental results in Section 5. Finally, Section 6 concludes the paper.

\section{Related Works}

Our proposed localization technique is range-free, which adopts similar assumptions as the existing three algorithms. In the following, we shall revisit the three existing range-free localization techniques in order to lay the foundation for the presentation of our proposed localization technique.

\subsection{DV-Hop Technique}

DV-hop technique [6] uses a mechanism that similar to the classical distance vector routing. The algorithm's implementation is comprised of three steps. First, each anchor broadcasts an announcement message to be flooded throughout the network, which contains the anchors ID, location and a hop-count parameter initialized to 0 . Each receiving node equipped with a counter maintains the minimum counter value of hops from itself to every anchor. When the announcement message received at a node, it will update hop-count and ignore the higher hop-count value received before, then forward the broadcast message to their neighbor nodes. Through this mechanism, every node in the network can get the shortest distance, hop-count value. In the second step, it estimates an average single hop distance, which is then broadcasted as a correction to the entire network. Finally, the unknown nodes compute their locations by multiplying the hopcount values with average hop distance. In the end of this step, once a node can calculate the distance to more than 3 anchors, it can use centroid formula to estimate its location notified with point. DV-Hop is defined as pointbased localization method because the output of localization is a point. This technique can produce a relatively high accuracy in networks where sensor nodes are evenly distributed and the objects to be tracked are static.

\subsection{Technique}

The Monte Carlo Localization (MCL) [7] is the first technique exclusively developed to track mobile sensor nodes. The algorithm calculates a set $L_{t}$ of $N$ location samples, each of which represents a possible location of the node to be tracked at time $t$. Initially, at $t=0$, MCL assumes that the node has no knowledge about its position; hence the first sample set $L_{0}$ consists of $N$ random samples which are selected within the deployment area. At each time step, the set $\left\{I_{t}^{i}\right\}$ is updated based on possible movement of the node and new observations on the node's connectivity to the anchor nodes. This process can be divided into two phases:

1) Prediction: In this phase, the node uses its previous location and maximum velocity, $v_{\max }$ to predict its possible new location. For example, if the node was at location $l_{t-1}^{i}$ at time $t-1$, its current location $l_{t}^{i}$ should be within a circle with radius $d_{\max }$ from $l_{t-1}^{i}$, where $d_{\max }$ is the maximum distance that a mobile node can move within each time interval. Hence, from the old sample $l_{t-1}^{i}$ the algorithm randomly selects a new sample $l_{t}^{i}$ within the circle centered at $l_{t-1}^{i}$ with radius $d_{\text {max }}$. By this way, from the previous sample set $L_{t-1}=\left\{l_{t-1}^{i}\right\}$, a new sample set $L_{t}=\left\{l_{t}^{i}\right\}$ can be predicted.

2) Filtering: In this phase, the node can eliminate some predicted samples obtained from the prediction phase based the connectivity between the node and the anchors which set up some space constrain to the node location. For example, if node $M$ can hear an anchor $A$, its location must be within a distance $r$ from $A$, where $r$ is the radio range of the node/anchor. All location samples which fall out of this area ought to be eliminated. Consequently, the number of valid samples may drop below $N$ due to elimination, hence re-sampling (repeating the prediction and filtering phase) is used to maintain $N$ location samples at each time step.

Finally, the estimated location of the node at time $t$ is the average of all $N$ sample values in the sample set $L_{t}$. 


\subsection{MCB Algorithm}

Monte-Carlo Localization Boxed (MCB) technique [8] was developed based on the MCL technique. The major difference between MCB technique and MCL is on how to withdraw a new sample. In the prediction phase of MCB, new location samples are generated based on the following information: (1) information about the anchors heard by the mobile node, (2) the maximum velocity $v_{\max }$ and (3) the node's previous location. This would significantly reduce size of the area from which the new samples are withdrawn, thus improving the efficiency of prediction phase. Consequently, MCB reduces the number of re-sample iterations and speeds up the convergence. It is necessary to review the approach used to determine the area $B$ from which location samples are withdrawn:

1) Initialization: At $t=0$, the node has no knowledge about its location. Let $B_{0}$ denote the initial 'anchor box' from which the first sample set $L_{0}$ is drawn.

If the node is not connected to any anchor,

$B_{0}=\left\{\left(0, x_{r}\right) ;\left(0, y_{r}\right)\right\}$ where $x_{r}$ and $y_{r}$ is the maximum $x$ and $y$ coordinate of the deployment area. The first sample set $L_{0}$ consists of $N$ samples selected randomly within the deployment area. Otherwise, $B_{0}$ is constructed from the location of all anchors that the node can communicate with:

$$
B_{0}=\left\{\left(x_{\min }, x_{\max }\right) ;\left(x_{\min }, x_{\max }\right)\right\}
$$

Let $\left(x_{j}, y_{j}\right)$ denote the coordinates of the anchor $j$ and $N_{a}$ denote the total number of anchors heard:

$$
\left\{\begin{array}{l}
x_{\min }=\max \left(x_{j}-r\right), x_{\max }=\min \left(x_{j}-r\right) \\
y_{\min }=\max \left(y_{j}-r\right), y_{\max }=\min \left(y_{j}+r\right) ; j=1 \ldots N_{a} .
\end{array}\right.
$$

2) At each time step $t$ : when there exists a previous sample set $L_{t-1}$ (i.e. the sample set is no longer empty as in Initialization), for each old sample $l_{t-1}^{i}$ from the old set $L_{t-1}$. We construct a square of size $2 d_{\max }$ centered at the old sample. This new box is built from each sample in the old set $L_{t-1}$ and is called a sample box:

$$
B_{t}^{i}=\left\{\left(x_{\min }^{i}, x_{\max }^{i}\right),\left(y_{\min }^{i}, y_{\max }^{i}\right)\right\}
$$

Let $\left(x_{t-1}^{i}, y_{t-1}^{i}\right)$ denote the coordinates in $l_{t-1}^{i}$, we have

$$
\left\{\begin{array}{l}
x_{\min }^{i}=\max \left(x_{\min }, x_{t-1}^{i}-d_{\max }\right), \\
x_{\text {max }}^{i}=\min \left(x_{\max }, x_{t-1}^{i}+d_{\max }\right), \\
y_{\text {min }}^{i}=\max \left(y_{\min }, y_{t-1}^{i}-d_{\max }\right), \\
y_{\text {max }}^{i}=\min \left(y_{\text {max }}, y_{t-1}^{i}+d_{\max }\right), i=1 \ldots N .
\end{array}\right.
$$

The area from which new samples are withdrawn would be the overlap of this square and the anchor box is shown in Figure 1.

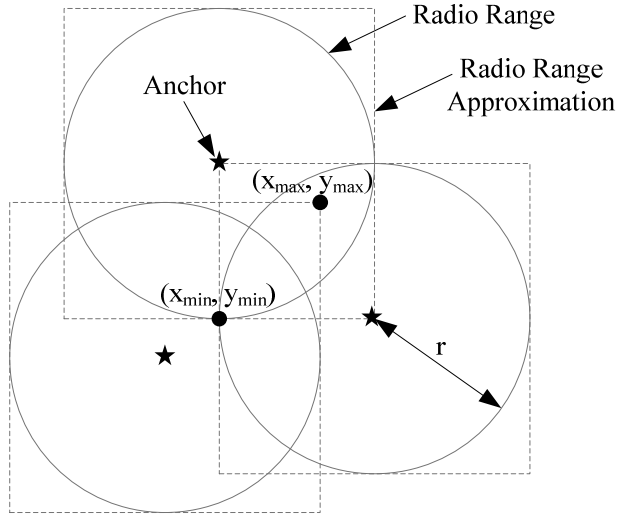

Figure 1. Determine Anchor Box [8].

\section{Proposed Localization Technique}

The proposed localization technique reduces computational complexity and improves efficiency by the two features: (1) Grid-based Output: The algorithm divides the monitored area into an $n \times n$ grid structure and generates an output region as the estimated node location; which may consist of one or many neighboring grids; (2) Event-triggering Mechanism: We proposed to adopt sleep -wake cycles for the localization module in the sensor nodes in order to save the scarce battery power of sensor nodes without sacrificing the algorithm's efficiency. In other words, the localization module does not operate continuously; instead, it is only activated whenever the temperature obtained by the sensing module exceeds a predefined threshold. Furthermore, the proposed localization technique adopts similar assumptions as the MCL and MCB algorithm, which are: (1) Anchor nodes, which are equipped with GPS or fixedly-placed at pre-known locations, are allowed to know their location all the time; (2) The transmission range of all anchor nodes is identical and equal to $R$.

\subsection{Proposed Algorithm}

The basic idea is to let the monitored area, e.g. the forest or nature reserve park, be bounded within $\mathrm{x}$-coordinates $\left(0, X_{s}\right)$ and $y$-coordinates $\left(0, Y_{s}\right)$. The algorithm first divides the area into an $n \times n$ grid structure and places four fixed anchor nodes at four corners, one mobile anchor (attached at a firefighting helicopter) with height $h$ moving in a certain trail whose projection is a circle including the center of the area, as shown in Figure 2. In particular, if the area is large or not of a square shape, we may repeat this arrangement over and place more anchors at corners of each $n \times n$ grid structure. For the sake of clarity, the smallest area unit is named as grid and each $n \times n$ grid structure as square. As shown in Figure 3 , on the ground plane, the dimension of each grid is 


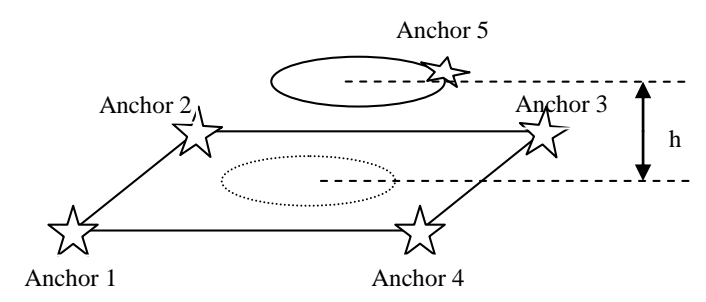

Figure 2. A square consists of four fixed anchors and one mobile anchor.

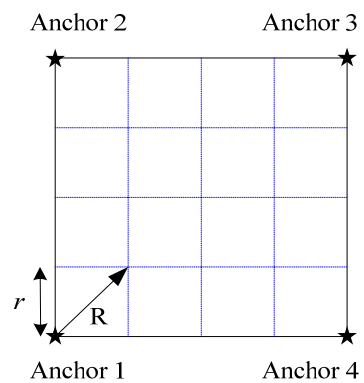

Figure 3. A square consisting of 16 grids in a $4 \times 4$ structure.

$r \times r$, where $r=R \times \cos 45^{\circ}$.

Similar to the DV-hop technique, our algorithm requires a set of distance information from a sensor node to each anchor node. Whenever the temperature sensed at a sensor node rises above a predefined threshold, the node would send a packet containing the set of hop-counts to the base station, which will be the inputs of the algorithm. The current set of hop-counts is

$H^{c}=\left\{h_{1}^{c}, h_{2}^{c}, \ldots, h_{N_{a}}^{c}\right\}$ where $h_{j}^{c}$ is current number of hops from the sensor node to anchor node $j$, and $N_{a}$ is the number of anchor nodes. The output of our algorithm is the estimated rectangular region-based location $B_{\text {est }}$ of node $M$ expressed in terms of left-bottom and right-top vertices' coordinates $\left(x_{\min }, y_{\min }\right)$ and $\left(x_{\max }, y_{\max }\right)$.

The tentative node location is determined based on $H^{c}$. For example, consider $N_{a}=5$ and

$H^{C}=\left\{h_{1}^{c}, h_{2}^{c}, h_{3}^{c}, h_{4}^{c}, h_{5}^{c}\right\}$, for each values $h_{j}^{c}$ in $H^{c}$, it is implied that the distance between the corresponding anchor and the node to be sensed is

$d \in\left[\left(h_{j}^{c}-1\right) \cdot R, h_{j}^{c} \cdot R\right]$. The algorithm estimates the location of sensor node $M$ as follows:

The algorithm determines the region $B_{j}^{c}$ which is the $h_{j}^{c}$-hop coverage area of anchor $A_{j}$ with coordinates $\left(X_{A j}\right.$, $Y_{A j}$ ), and $B_{j}^{c}$ is a square with side length $h_{j}^{c} r$. The upper-bound and lower-bound for $x$ and $y$ coordinates of $B_{j}^{c}$ are:

$$
\left\{\begin{array}{l}
x_{j, \text { min }}^{c}=\max \left\{0, X_{A_{j}}-r h_{j}^{c}\right\} \\
x_{j, \text { max }}^{c}=\min \left\{X_{r}, X_{A_{j}}+r h_{j}^{c}\right\} \\
y_{j, \text { min }}^{c}=\max \left\{0, Y_{A_{j}}-r h_{j}^{c}\right\} \\
y_{j, \text { max }}^{c}=\min \left\{Y_{r}, Y_{A_{j}}+r h_{j}^{c}\right\}
\end{array}\right.
$$

For the mobile anchor above the area, only its projection is focus, and the trail will be a square shaped instead of circle in order to simplify the process. The hop count is also obtained based on its projection. The 3-D to 2-D conversion is accomplished as shown in Figure 4.

Next, the algorithm finds the overlap of all regions, i.e., $B_{j}^{c}$, where $j$ is $1,2, \ldots, N_{a}$. Then,

$$
B^{c}=B_{1}^{c} \cap B_{2}^{c} \cap \cdots \cap B_{N_{a}}^{c}
$$

The region $B^{c}$ would be the tentative estimated grid -based location of sensor node $M$. The upper-bound and lower-bound for $x$ and $y$ coordinates of this rectangular region are:

$$
\begin{aligned}
& \left\{\begin{array}{l}
X_{\text {min }}^{c}=\max \left\{X_{1, \text { min }}^{c}, X_{2, \text { min }}^{c}, \ldots, X_{N_{a}, \text { min }}^{c}\right\} \\
X_{\text {max }}^{c}=\min \left\{X_{1, \text { max }}^{c}, X_{2, \text { max }}^{c}, \ldots, X_{N_{a}, \text { max }}^{c}\right\}
\end{array}\right. \\
& \left\{Y_{\text {min }}^{c}=\max \left\{y_{1, \text { min }}^{c}, y_{2, \text { min }}^{c}, \ldots, y_{N_{a}, \min }^{c}\right\}\right. \\
& Y_{\text {max }}^{c}=\min \left\{y_{1, \text { max }}^{c}, y_{2, \text { max }}^{c}, \ldots, y_{N_{a}, \text { max }}^{c}\right\}
\end{aligned}
$$

As shown in Figure 5, $B^{c}$ could be either a single grid, e.g., for input $H^{c}=\{3,3,2,3,2\}$ in Figure 5(a) or larger grid, e.g., for $H^{c}=\{2,0,0,0,3\}$ in Figure 5(b).

If the resulted $B^{c}$ is null, the algorithm will return the entire monitored area as an output, i.e.,

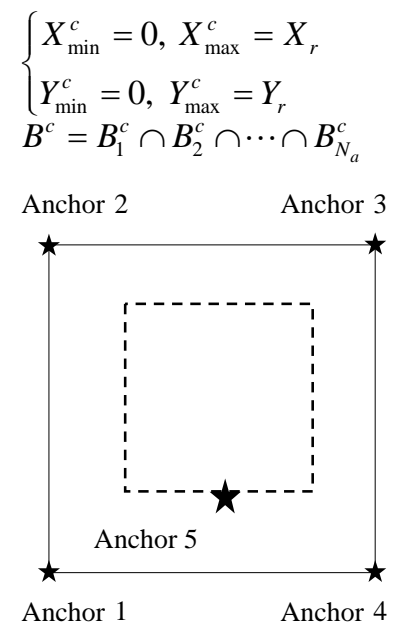

Figure 4. 2-D model with 4 fixed anchors and 1 mobile anchor.

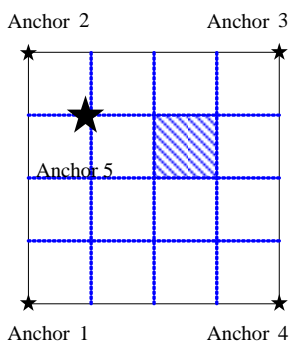

(a)

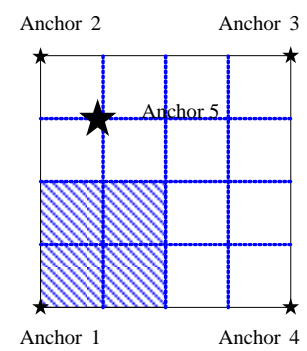

(b)
Figure 5. Estimated region for node $M$ for (a) $H^{c}=$ $[3,3,2,3,2] ;$ (b) $H^{c}=[2,0,0,0,3]$. 
Besides the coordinates of each anchor, the height $h$ of the mobile anchor is also a significant variable retrieved from the sensors. In the real implementation, as the height increases, a larger transmission power is required in order to communicate with the on-ground node to be sensed. As shown in Figure 6, the target node $t$ is at point $C$, the mobile anchor with height $h$ is at point $A$, and the projection of the mobile anchor is at point $\mathrm{B}$. If the minimum transmission power used to communicate between point $\mathrm{B}$ and point $\mathrm{C}$ is $P_{B}$, the received power, $P_{C}$, at point $\mathrm{C}$ is given by

$$
P_{C}=P_{B} \times d^{-n}
$$

where $d$ is the distance between points $\mathrm{B}$ and $\mathrm{C}$ and $\mathrm{n}$ is the path-loss exponet. Since the mobile anchor is at point A, a larger transmission power, $P_{A}$, at point $A$ is required so that at point $\mathrm{C}$,

$$
P_{C}=P_{A} \times l^{-n}=P_{A} \times\left(\sqrt{h^{2}+d^{2}}\right)^{-n}
$$

Using (9) and (10), the required transmit power, $P_{A}$, at point $\mathrm{A}$ is

$$
P_{A}=\frac{P_{B} \times\left(\sqrt{h^{2}+d^{2}}\right)^{n}}{d^{n}}
$$

From (11) we can see that $h$ is important because $P_{A}$ is increases with $h$. Thus, during the real implementation, $P_{A}$ should be adjusted based on $h$.

\subsection{Proposed Algorithm vs. DV-Hop}

Our proposed algorithm as introduced in Section 3.1 has several common ideas with the DV-Hop algorithm introduced in Section 2.1. The comparisons are stated in this section. Firstly, although our proposed algorithm requires input similar to DV-Hop including a set of hop -count unit from one node to each anchor, the estimate hop distance is no longer needed and in addition, the calculation of centroid formula is also unnecessary. We assume the monitoring area, e.g. certain forest region, can be divided into an n-by-n grid structure, where the diagonal of grid is equal to the transmission rang of

A

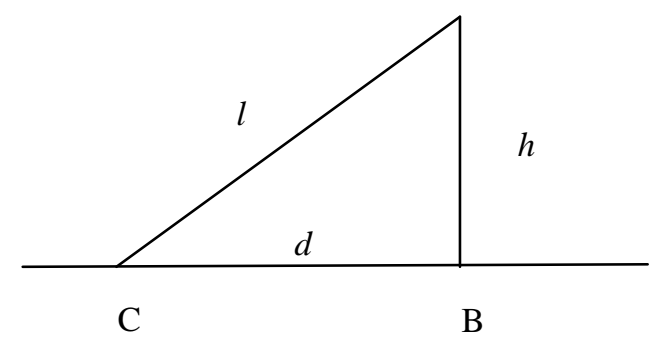

Figure 6 Illustration graph with mobile anchor at A, node to be sensed at $C$. every anchor. Therefore, the hop distance is an identical constant value as well as one hop is the same as one grid. And then, the algorithm's output will be a region consisting of integer number of grids. It's obviously that the grid-based localization process and output are different from DV-Hop's which needs less calculation.

Moreover, nodes in the network have limited energy resources and weak processing capabilities. If the algorithm uses most of a sensor's energy to locate itself, it will have no more left to perform tasks. On the other hand, considering many applications for wild environment which is hard to replace the battery of sensors, the sensors should service as long as possible for the optima utilization. Therefore, minimizing the power consumption of sensors is needed. As compared to the pointbased localization, grid-based localization is less complicated yet with less processing time, thus it reduces calculations, saves power consumption and considerably enhances the sensor's lifetime.

\section{Simulation Results}

The proposed localization algorithm under the condition of 4 fixed anchors and 1 mobile anchor (called mobile anchor model) as well as only 4 fixed anchors (called basic model) is simulated in Matlab environment. Accuracy test results, location test results and the comparison results between basic model and the mobile anchor model will be illustrated in the followings.

\subsection{Accuracy Test}

The key performance metric of localization algorithms is the output accuracy. This test is to prove that the output region can always cover the target node. During the simulation process, many sample nodes are randomly generated, labeled in green color, and through the algorithm proposed, the corresponding outputs will be labeled out in red color. Figure 7 shows 2 of the accuracy test results under mobile anchor model at $n=10$.

Through the simulation result, the accuracy of the algorithm is $100 \%$ which implies that the estimated outputs can always cover the targeting nodes. Even in the real implementation, due to the environmental effect, sometimes the transmission range might be less than the
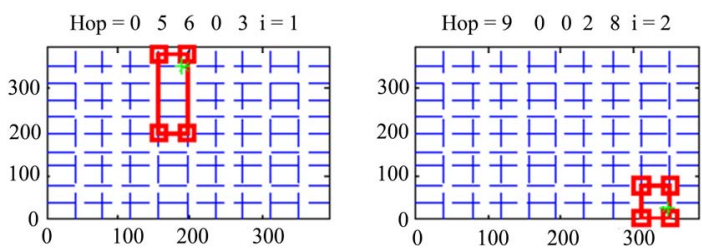

Figure 7. Accuracy test results at $\boldsymbol{n}=10$ (2 samples are displayed). 
default value, the accuracy of the algorithm is still 100\%. The reason is when the transmission range is a smaller value, the hop count might be change to a larger value, and thus, the estimated output region will be slightly larger than the simulation result, but it still can cover the targeting node. So, the algorithm always performs a $100 \%$ detection.

\subsection{Location Test}

The location test investigates the size of the estimated output region for a target node on different locations over the monitored area. The test randomly generates 2000 node-location samples with coordinates $(x, y)$ within the monitored area with $x \in\left[0, X_{r}\right], y \in\left[0, Y_{r}\right]$. The monitored area is divided into a $10 \times 10$ grid structure. Each node-location $(x, y)$ with respect the four anchor nodes is then transformed to the current hop-count values, which serve as the input for our algorithm. The test subsequently estimates the grid-based location for each sample using our algorithm, calculates the area of the estimated region in grid unit, and plots the estimated output region's area as a function of its node-location as shown in Figures 8 and 9.

In Figure 8, it only consists of four fixed anchors at

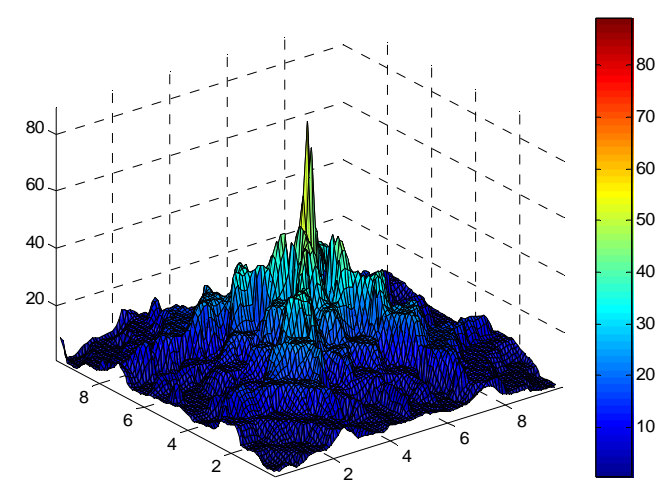

Figure 8. Location test of $\mathbf{4}$ fixed anchors (basic model).

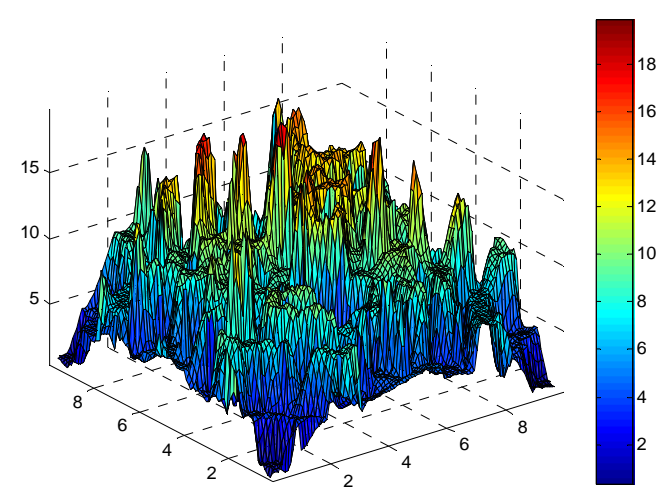

Figure 9. Location test of 4 fixed anchors and 1 mobile anchor (mobile anchor model). the corner of the monitoring area whereas in Figure 9, the result is obtained under the mobile anchor model which introduces one more mobile anchor moving around the center. It is observed that under the mobile anchor model, the maximum size of the output region is 18 to 20 grids and the average output is around 10 grids. While in the basic model, the maximum size of the output region reaches 80 to 90 grids at the center and the average output size is around 40 grids. In general, the output is desirable to be a region with as small as possible area to quickly locate the fire's actual position. Therefore, the smaller the estimated region's area is, the better the algorithm's accuracy is. It is clear that after adding the mobile anchor, the size of the output region reduced from $40 \%$ to $10 \%$ on average, and the peak outputs at the center are also diminished, such that an equally distributed size of the output regions are generated.

\subsection{Performance Comparison between Basic Model and Mobile Anchor Model}

In this test, we study the performance between basic model and mobile anchor model in terms of the probabilities of outputting different sizes of regions. The CDF curves are plotted for these two models as shown in Figures 10 and 11. In each test, the size of the monitoring area is fixed, and 5000 node-location samples are randomly generated. Figure $\mathbf{1 0}$ shows the results when the monitoring area is a 10-by-10 grid structure and Figure 11 shows the result when the area is a 12-by-12 grid structure. The $\mathrm{x}$-axis indicates the output size and the $y$-axis corresponds to the probabilities of outputting such region size.

From the result it can be seen that the curves for the mobile anchor model are always higher than that of the basic model, which implies that the addition of this mobile anchor increases the probability of outputting a

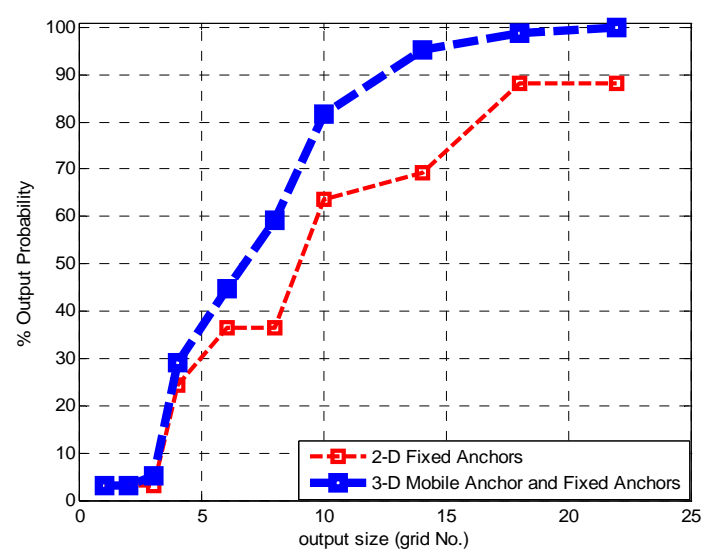

Figure 10. CDF Curves at $\boldsymbol{n}=\mathbf{1 0}$. 


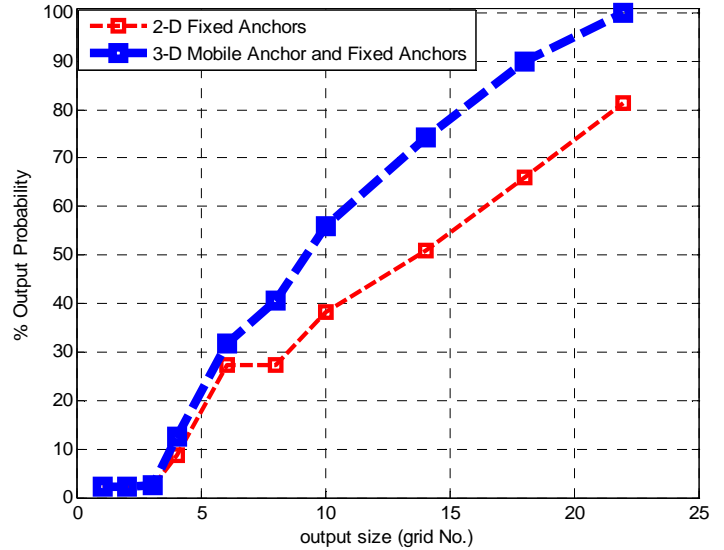

Figure 11. CDF Curves at $n=12$.

small size region and improves the performances of the algorithm.

As for a large size of the monitoring area, the probability of outputting of a region with grid size less than 5 is very small for both models. However, as the output grid size increases, the performance of mobile anchor model performs better than basic model. In fact, the mobile anchor improves the algorithm more for a larger monitoring area. This is also observable if the comparison is done between them as shown in the Figures, in which the tendencies of the two curves go further away as $n$ increases to 12 .

\section{Experiment Results}

Two experiments have been conducted to test the proposed localization technique. As shown in Figure 12, the two experiments were implemented in the soccer field with a square of size $60 \mathrm{~m} \times 60 \mathrm{~m}$.

The monitoring area was divided into $3 \times 3$ grids so that, each grid occupies a $20 \mathrm{~m} \times 20 \mathrm{~m}$ area. The transmission power of each sensor node was set as $-1 \mathrm{dBm}$ for which, the radio transmission range could reach around $30 \mathrm{~m}$, which was approximately equal to the diagonal length of each grid. Four Anchors were placed at the four corners of the region and four relay nodes were placed at the four points indicated as A, B, C, and D. To simulate the animals' motion, an experimenter holding the sensor node was moving inside the region from the grid marked “ 1 ", “ 2 " “3”, and until the middle grid marked " 9 ”. At the base station side which was connected to a PC terminal, the monitoring software would analyze the data sent by the sensor nodes and then output the grid region which the examiner was in.

\subsection{Hardware Requirements}

Table 1 shows the hardware list during the implementa

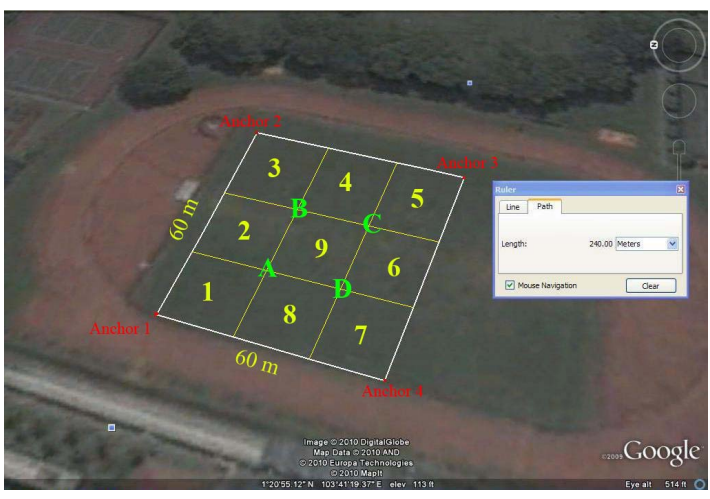

Figure 12. Monitoring Area Layout.

Table 1. Hardware List.

\begin{tabular}{cc}
\hline Hardware & Model \\
\hline $\begin{array}{c}\text { Gateway board } \\
\text { Mote sensor data acqui- } \\
\text { sition board }\end{array}$ & MB520 \\
Mote & MPR2400CA \\
Workstation & Any model
\end{tabular}

tion. For further information about each hardware component, Interested readers can refer to the Crossbow MICAz datasheet [9], MPR-MIB Users Manual [10] and MTS/MDA Sensor Board Users Manual [11].

\subsection{Results for Experiment 1}

The experiment started as the examiner walking from Anchor 1. Under this condition, the node held by the examiner could communicate with Anchor 1 directly, communicate with Anchor 2 through relay nodes $\mathrm{A}$ and $\mathrm{B}$, communicate with Anchor 3 through relay nodes $\mathrm{C}$ and $\mathrm{A}$, and communicate with Anchor 4 through relay nodes $\mathrm{D}$ and $\mathrm{A}$. So the set of hop-counter values received at base station was $H=\{1,3,3,3\}$. By using the formula $B^{c}=B_{1}^{c} \cap B_{2}^{c} \cap \cdots \cap B_{N_{a}}^{c}$ to calculate the overlap area, the output was then generated and as shown in Figure 13.

Next, when the examiner reached the border of grid 1 and grid 2, where the hop-counter value was $H=\{1,2,3$, 3 , the system would output a line in between those two grids, which represented the overlapped region as shown in Figure 14.

As the process continued, the examiner would reach purely inside grid 2, so that, the set of hop counters would be $H=\{2,2,3,3\}$. But by using the formula $B^{c}=B_{1}^{c} \cap B_{2}^{c} \cap \cdots \cap B_{N_{a}}^{c}$ to calculate the overlap region, the output would be a two-grid sized region which is 


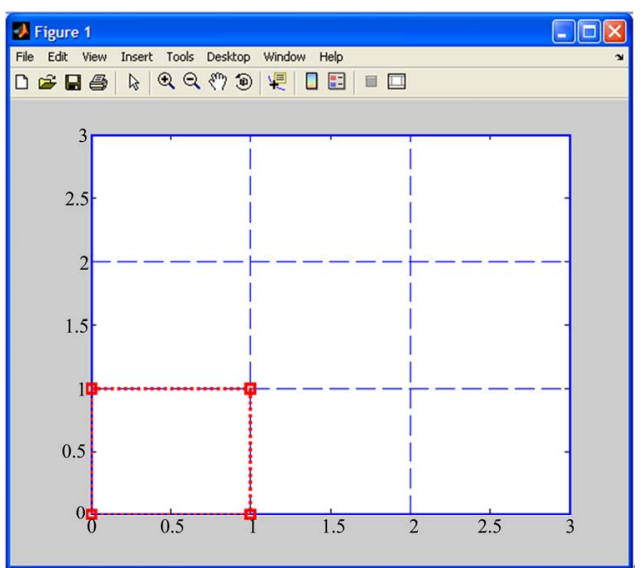

Figure 13. $H=\{1,3,3,3\}$.

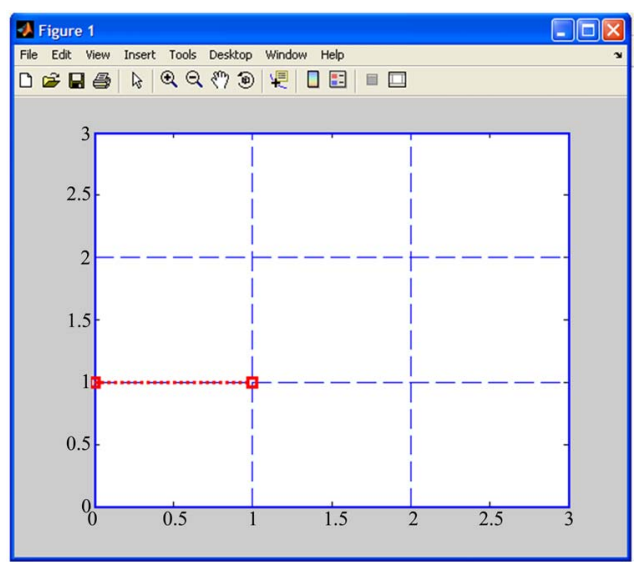

Figure 14. $H=\{1,2,3,3\}$.

shown in Figure 15.

The output size in this situation was increased to 2 grids, which caused the decrease of the localization accuracy. The second experiment would show the proposed method to improve accuracy, and it will be shown in detail later in this Section.

Continue with the current experiment, when the examiner reached the border of grid 2 and grid 3, where the set of hop counters was $H=\{2,1,3,3\}$, the output is shown in Figure 16.

Then, Figure 17 shows the output region whe $\mathrm{n}$ the examiner was purely inside the grid 3 and $H=\{3,1,3$, $3\}$.

The experiment continued until the examiner reached the middle grid where the set of hop counters received was $H=\{2,2,2,2\}$. Figure 18 shows the output result.

\subsection{Results for Experiment 2}

With all the other conditions no change, experiment 2 was implemented with one modification, which was to introduce a mobile Anchor. As the simulation results

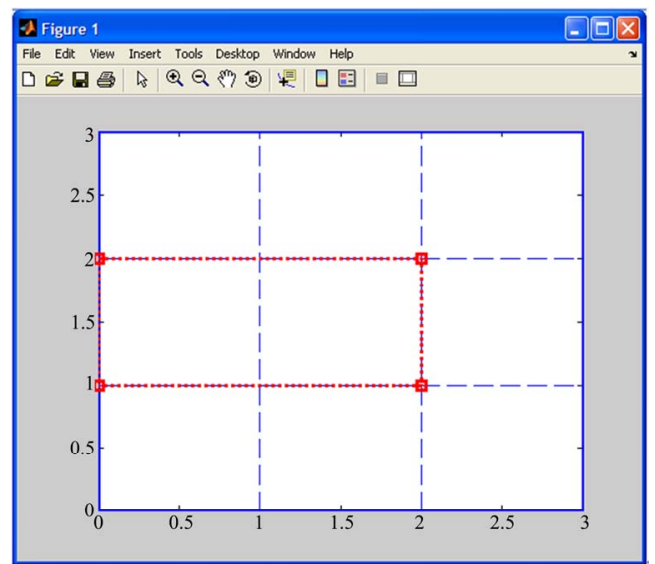

Figure 15. $H=\{2,2,3,3\}$.

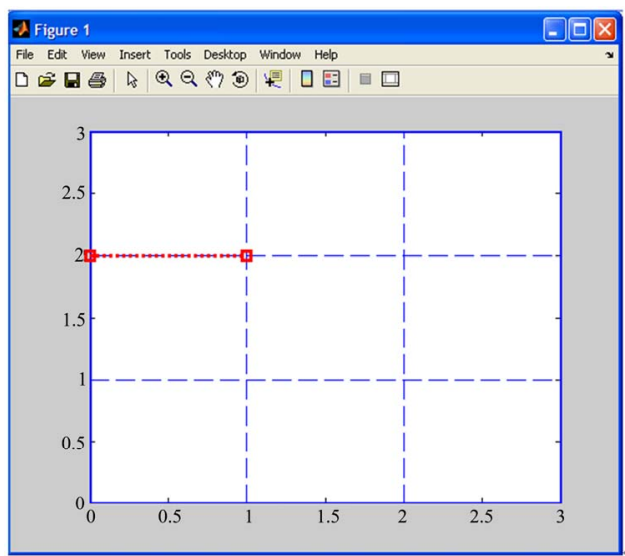

Figure 16. $H=\{2,1,3,3\}$.

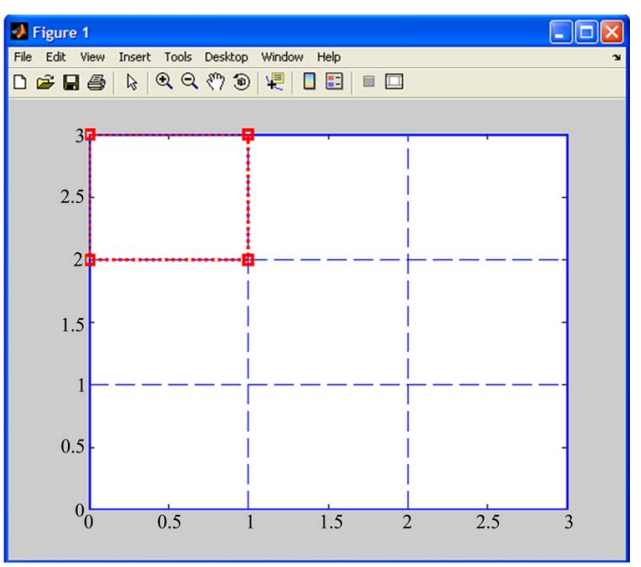

Figure 17. $H=\{3,1,3,3\}$.

showed in the previous section, by adding a 3-D mobile Anchor, the accuracy of localization could be largely increased, as shown in Figure 8 to Figure 9. For convenient implementation, this experiment 2 only used the projection of the 3-D mobile anchor trail as the route.

The added mobile Anchor was carried by a remote 


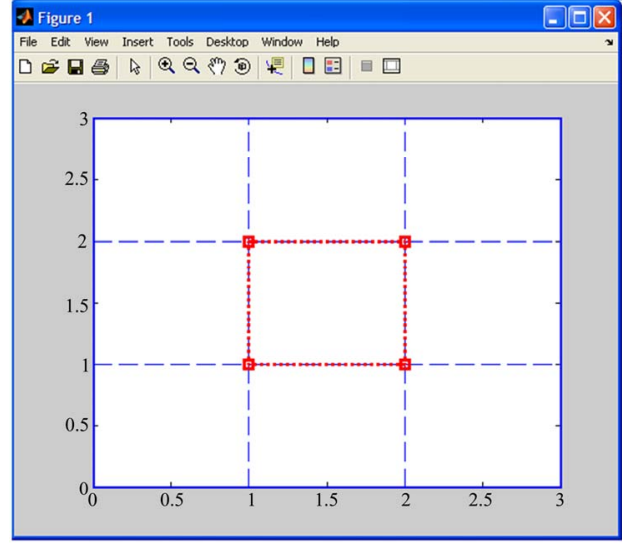

Figure 18. $H=\{2,2,2,2\}$

control car and moving inside the region along the path A-B-C-D as shown in Figure 12.

First, if examiner holding the node was at the location indicated by "E" as shown in Figure 19, where the hop-counter values was $H=\{2,2,3,3\}$, the algorithm without mobile anchor could only calculate out the result as shown in Figure 20. Then for the case with mobile Anchor, if the mobile Anchor was moving near the location "C" or " $\mathrm{D}$ " and broadcasting a message, this message would be relayed by one node either located at " $\mathrm{A}$ " or " $\mathrm{B}$ " and then received by the node. The base station could then analyze this message, as long as the message was not directly received by the node, the base station would know that, the node was not within one grid distance from the mobile Anchor, so that, grid 9 would be filtered out, and the grid 2 would be the final output as shown in Figure 21.

But the point needs to be noticed is that this mobile Anchor method is not able to filter out the unwanted grid every time. For example, if the examiner holding the node was still inside grid 2, but at the same time, the mobile Anchor was moving near to location "A" and "B", then the node could communicate with the mobile Anchor directly which meant that, the node was within one grid distance away from the mobile Anchor. After this information was known by base station, it could not do further analysis because no matter the node was inside grid 2 or grid 9 could both communicate with the mobile Anchor directly. So as a conclusion, the possibility for filtering unwanted grid in this condition could reach up to $50 \%$.

The $50 \%$ possibility in this implementation is large enough to be accepted, because this implementation experiment is a very basic model $(3 \times 3$ grid, small monitoring area), and it is only aimed to verify the availability of the mobile Anchor method. As proved by the simulation results showed in the previous section, if the mobile Anchor is introduced to a much larger monitoring area, it

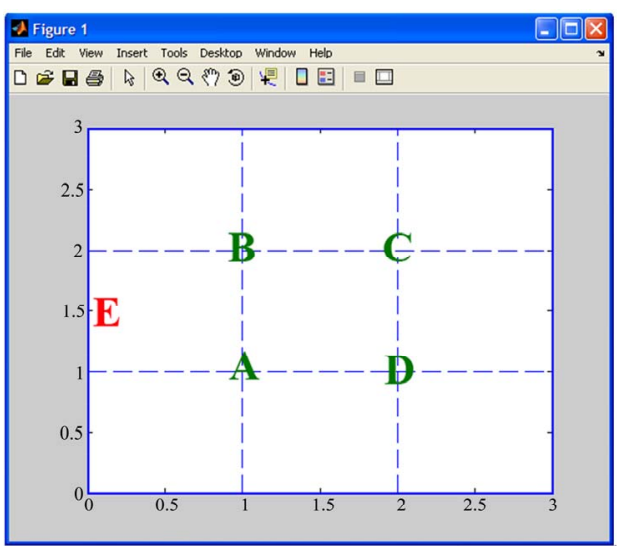

Figure 19. The Location of Examiner.

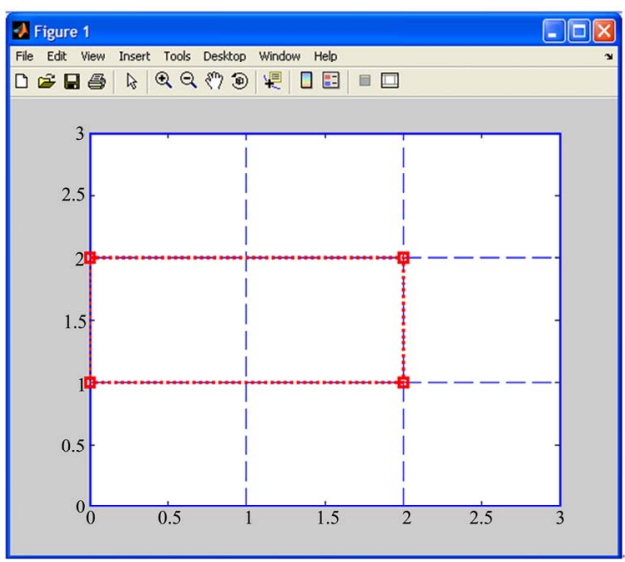

Figure 20. $H=\{2,2,3,3\}$ without Mobile Anchor.

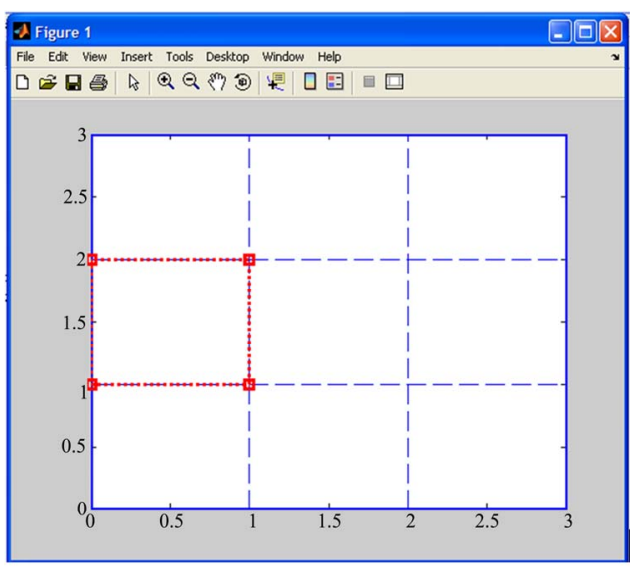

Figure 21. $H=\{2,2,3,3\}$ with Mobile Anchor.

will be more efficient.

\section{Conclusions}

This paper presents a localization technique particularly tailored for forest fire surveillance systems. By adopting grid-based output and event-triggering mechanism, the 
proposed algorithm can reduce computational complexity, improve output accuracy and reduce power consumption. Computer simulations were conducted to verify that the proposed algorithm is efficient. Furthermore, two implementation experiments have been conducted with the proposed algorithm. For the first experiment without mobile Anchor, $66.67 \%$ of the scenarios could return single-grid location estimation. The second experiment with mobile Anchor would further increase the accuracy by $50 \%$. Experimental results have shown that the proposed algorithm reduces the complexity of implementation, and it can provide considerable accuracy.

\section{References}

[1] G. Mao, B. Fidan and B. D. O. Anderson, "Wireless Sensor Network Localization Techniques," Computer Networks, Vol. 51, No. 11, July 2007, pp. 2529-2553.

[2] P. Bahl and V. N. Padmanabhan, "RADAR: an in-building RF-based user location and tracking system," in Proceedings of IEEE/ACM INFOCOM'00, Tel Aviv, Israel, Vol. 2, 26-30 March 2000, pp. 775-784.

[3] A. Ward, A. Jones and A. Hopper, "A New Location Technique for the Active Office," IEEE Personal Communications, Vol. 4, No. 5, October 1997, pp. 42-47.
[4] D. Niculescu and N. Badri, "Ad hoc positioning system (APS) using AOA," in Proceedings of IEEE/ACM INFOCOM'03, San Francisco, CA, Vol. 3, 30 March-3 April 2003, pp. 1734-1743.

[5] N. B. Priyantha, A. Chakraborty and H. Balakrishnan, "The Cricket Location-Support System," in Proceedings of ACM MobiCom'00, Boston, MA, Vol. 1, 2000, pp. 32 $-43$.

[6] D. Niculescu and B. Nath, "Ad Hoc Positioning System (APS)," in Proceedings of IEEE GLOBECOM '01, San Antonio, TX, Vol. 5, 25-29 November 2001, pp. 2926 2931.

[7] L. Hu and D. Evans, "Localization for Mobile Sensor Networks," in Proceedings of ACM MobiCom'04, Philadelphia, PA, Vol. 1, 26 September-1 October 2004, pp. 45-57.

[8] A. Baggio and K. Langendoen, "Monte-Carlo Localization for Mobile Wireless Sensor Networks," Ad Hoc Networks, Vol. 6, No. 5, January 2008, pp. 718-733.

[9] Crossbow Technology, “MICAz,” 2007.

[10] Crossbow Technology, “MPR-MIB Users Manual,” 2007.

[11] Crossbow Technology, "MTS/MDA Sensor Board Users Manual," 2007. 